\title{
INITIAL CHECKOUT RESULTS OF THE COMPACT INFRARED CAMERA (CIRC) FOR EARTH OBSERVATION
}

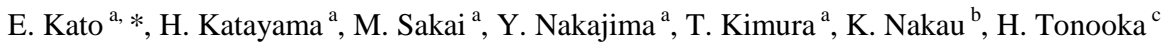 \\ a Japan Aerospace Exploration (JAXA), Satellite Applications Mission Directorate I, Satellite Technology Innovation Office, Sensor \\ System Research Group, Ibaraki, Japan - (katoh.eri, katayama.haruyoshi, sakai.michito, nakajima.yasuhiro, \\ kimura.toshiyoshi)@jaxa.jp \\ ${ }^{\mathrm{b}}$ Hokkaido University, Hokkaido, Japan - knakau@ sci.hokudai.ac.jp \\ c Ibaraki University, Ibaraki, Japan - tonooka@mx.ibaraki.ac.jp
}

\section{Commission}

KEY WORDS: thermal infrared imaging, uncooled infrared array detector, wildfire, ALOS-2, JEM-CALET

\begin{abstract}
:
Compact Infrared Camera (CIRC) is a technology-demonstration instrument equipped with an uncooled infrared array detector (microbolometer) for space application. CIRC is the first microbolometer sensor without a calibration function in orbit, like a shutter system or an onboard blackbody. The main objective of the CIRC is to detect wildfires, which are major and chronic disasters affecting various countries of Southeast Asia, particularly considering the effects of global warming and climate change. The CIRC achieves a small size (approximately $200 \mathrm{~mm}$ ), light mass (approximately $3 \mathrm{~kg}$ ), and low electrical power consumption $(<20 \mathrm{~W}$ ) by employing athermal optics and a shutterless system. The CIRC can be consequently mounted on multiple satellites to enable highfrequency observation. Installation of CIRCs on the ALOS-2 and on the JEM/CALET is expected to increase observation frequency. We present the initial check-out results of the CIRC onboard ALOS-2. Since the initial check-out phase (July 4-14, 2014), the CIRC has acquired the images of Earth. CIRC was demonstrated to function according to its intended design. After the early calibration validation phase, which confirmed the temperature accuracy of observed data, CIRC data has been available to the public January 2015 onward. We also introduce a few observational results about wildfire, volcanoes, and heat-island.
\end{abstract}

\section{INTRODUCTION}

\subsection{Outline}

We have developed the Compact Infrared Camera (CIRC) to demonstrate microbolometer technology to thermal infrared imaging from space as described by (Katayama et al., 2009), (Kato et al., 2012), (Nakamura et al., 2013), and (Kato et al., 2014). The main objective of the CIRC is to detect wildfires, which are a major and chronic disaster that affects many countries, particularly those in the Asia-Pacific region. There are research studies suggesting that global warming and climate change may accelerate the severity of wildfires and other natural disasters. Their early detection is important. An effective means of early detection is to increase their observation frequency. Our aim is to realize frequent observations by loading CIRCs in as many satellites as possible by exploiting their small size, light mass, and low power consumption. Other mission targets of the CIRC include the observation of volcanoes and urban heat islands.

\subsection{ALOS-2 and JEM-CALET}

The CIRC will be carried as a technology demonstration payload on board the Advanced Land Observing Satellite-2 (ALOS-2) (see (Suzuki et al., 2009)) and the CALorimetric Electron Telescope (CALET) (see (Torii et al., 2008)) which will be attached to the Japanese Experiment Module (JEM-EF) an the International Space Station (ISS). ALOS-2 was launched in May 24, 2014, and JEM-CALET will be launched in 2015. This paper shows initial check results of the CIRC onboard ALOS-2 on the initial check-out phase.

The orbits of ALOS-2 and ISS are shown in Figure 1. The green lines represent the ALOS-2 orbit; this is synchronous subrecurrent orbit at an altitude of $628 \mathrm{~km}$. In contrast, the white lines represent the ISS orbit. It has an orbital inclination of 52.8 degree; it is located at an altitude of about $400 \mathrm{~km}$.

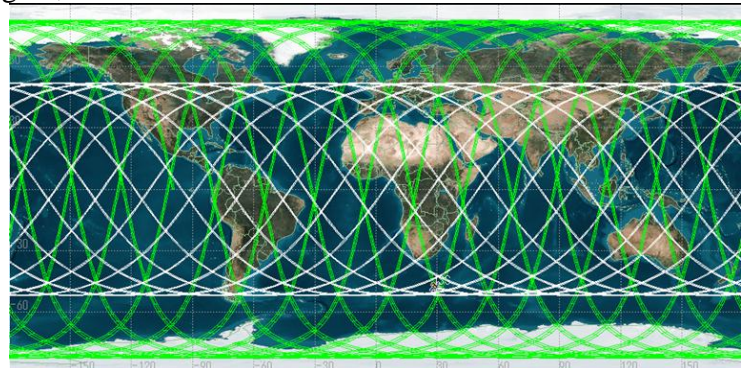

Figure 1. Orbit of ALOS-2 and ISS

\section{COMPACT INFRARED CAMERA (CIRC)}

\subsection{Specification of CIRC}

The main objective of the CIRC is to demonstrate the technology for detecting wildfires using a microbolometer. The CIRC includes athermal optical and shutterless systems. Owing to athermal optics, the camera's temperature does not need to be

\footnotetext{
* Corresponding author. This is useful to know for communication with the appropriate person in cases with more than one author.
} 
controlled using a heater. Thus, we realized a CIRC with a small size, light mass, and low power consumption.

The baseline specifications of the CIRC are listed in Table 1 . We set the baseline specifications such as spatial resolution and dynamic range (see Katayama et al., 2008). The detector has a large format $(640 \times 480$ pixels $)$ to obtain a wide field of view. Spatial resolution is an important factor for wildfire detection. The spatial resolution is $200 \mathrm{~m}$ from an altitude of $600 \mathrm{~km}$ (ALOS-2) and $130 \mathrm{~m}$ from an altitude of $400 \mathrm{~km}$ (CALET). Eliminating the cooling system reduces the size $(110 \mathrm{~mm} \times 180$ $\mathrm{mm} \times 230 \mathrm{~mm})$ and electrical power consumption $(<20 \mathrm{~W})$.

\subsection{CIRC onboard ALOS-2}

The CIRC onboard ALOS-2 takes images of the target area when the SAR is pointing to the right at an off-nadir angle of $30^{\circ}$. The mounting location of the CIRC is shown in Figure 2.

\begin{tabular}{|l|l|}
\hline Parameter & Specification \\
\hline Size & $110 \mathrm{~mm} \times 180 \mathrm{~mm} \times 230 \mathrm{~mm}$ \\
Mass & $3 \mathrm{~kg}$ \\
Detector & Uncooled infrared detector \\
Wavelength & $8-12$ micro meters \\
Number of pixels & $640 \times 480$ \\
Spatial resolution & $<200 \mathrm{~m} @ 600 \mathrm{~km}$ (ALOS-2) \\
& $<130 \mathrm{~m} @ 400 \mathrm{~km}$ (CALET) \\
Field of view & $12^{\circ} \times 9^{\circ}$ \\
Exposure & $33 \mathrm{~ms}$ \\
Dynamic range & $180-400 \mathrm{~K}$ \\
Bit number & $14 \mathrm{bits}$ \\
NEDT & $0.2 \mathrm{~K} @ 300 \mathrm{~K}$ \\
FPN & $0.3 \mathrm{~K} @ 300 \mathrm{~K}$ \\
Temperature accuracy & $4 \mathrm{~K}$ (goal: $2 \mathrm{~K} @ 300 \mathrm{~K})$ \\
\hline
\end{tabular}

Table 1. Specifications of CIRC
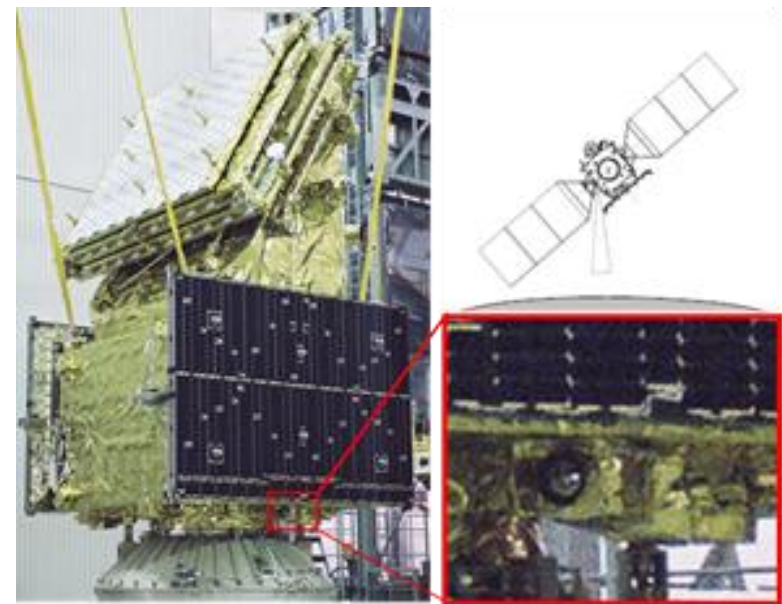

Figure 2. Mounting location of the CIRC

\section{ALOS-2/CIRC BASIC OBSERVATION PLAN}

In stationary operation phase, we plan to observe regions according to three missions, wildfire, volcanoes, and urban heat island, based on ALOS-2/CIRC basic observation plan.

As for wildfire, observation regions are decided as those of the top $10 \%$ of the monthly average of MODIS Hotspots during a past decade. CIRC observes monthly different wildfire-prone areas. In addition, thirty active volcanoes both domestically and abroad are chosen, and observed year-round. About urban heat island, major cities in Japan and Asia region are selected as basic observation region. CIRC also observes land area including Japanese remote island and calibration sites needed for stationary monitor.

Figure 3 shows ALOS-2/CIRC basic observation plan in January and July. CIRC observes different regions varying with areas and climate on a month-by-month basis because wildfire generally occurs in a dry season. Figure 4 shows ALOS-2/CIRC basic observation plan in September and planning example of a recurrence cycle (14 days) based on the basic observation plan. About half of basic observation plan region is available to be covered in a recurrence cycle although observable region is actually affected by ALOS-2/PALSAR-2 observation plan. Some areas are also observable more than once.

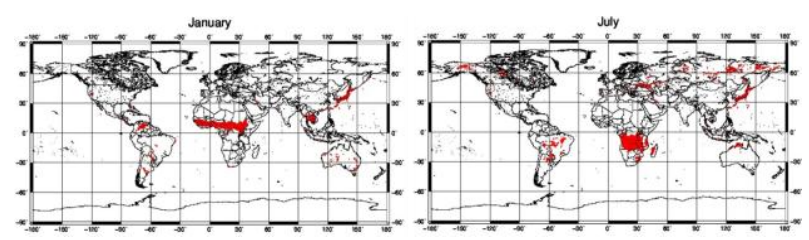

Figure 3. ALOS-2/CIRC basic observation plan in January and July

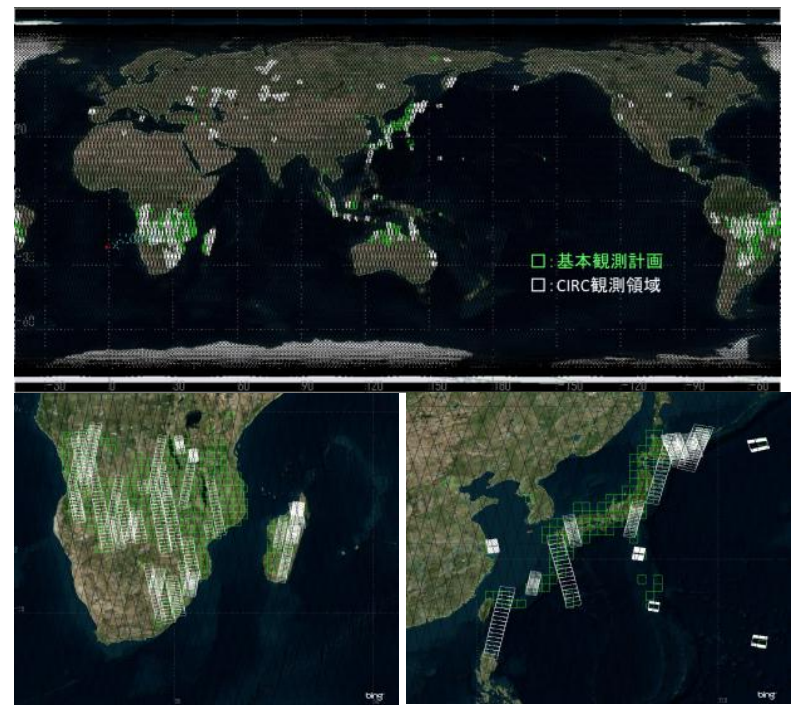

Figure 4. ALOS-2/CIRC basic observation plan in September (Green Box) and planning example of a recurrence cycle (White Box).

\section{INITIAL CHECK-OUT PHASE}

During the initial check-out phase of ALOS-2/CIRC from July 4 th to 14 th of 2014 , following items were verified:

1. CIRC basic functional

2. Performance in operation sequence (e.g., quality of images taken by CIRC)

3. Accuracy in pointing determination

In the initial check-out phase, telemetry was examined to confirm that command transmission and imaging function for actual operation sequence were properly working. Accuracy in pointing determination was examined from the image.

Figure 5 shows temperature of DC-DC converter and detector package during the initial check-out phase. The temperature fluctuation in orbit is from 10 to 12 degrees Celsius in non- 
operating state although CIRC does not have temperature control function. CIRC is set in an extremely stable environment relative to CIRC operating temperature range (-15 to 50 degree). When power of the CIRC is turned on, temperature in both parts increases in the same way as ground test. CIRC is possible to start next observation without temperature rise in forty minutes (thirty minutes of those is for temperature stabilization) per an orbit which is current CIRC Duty.
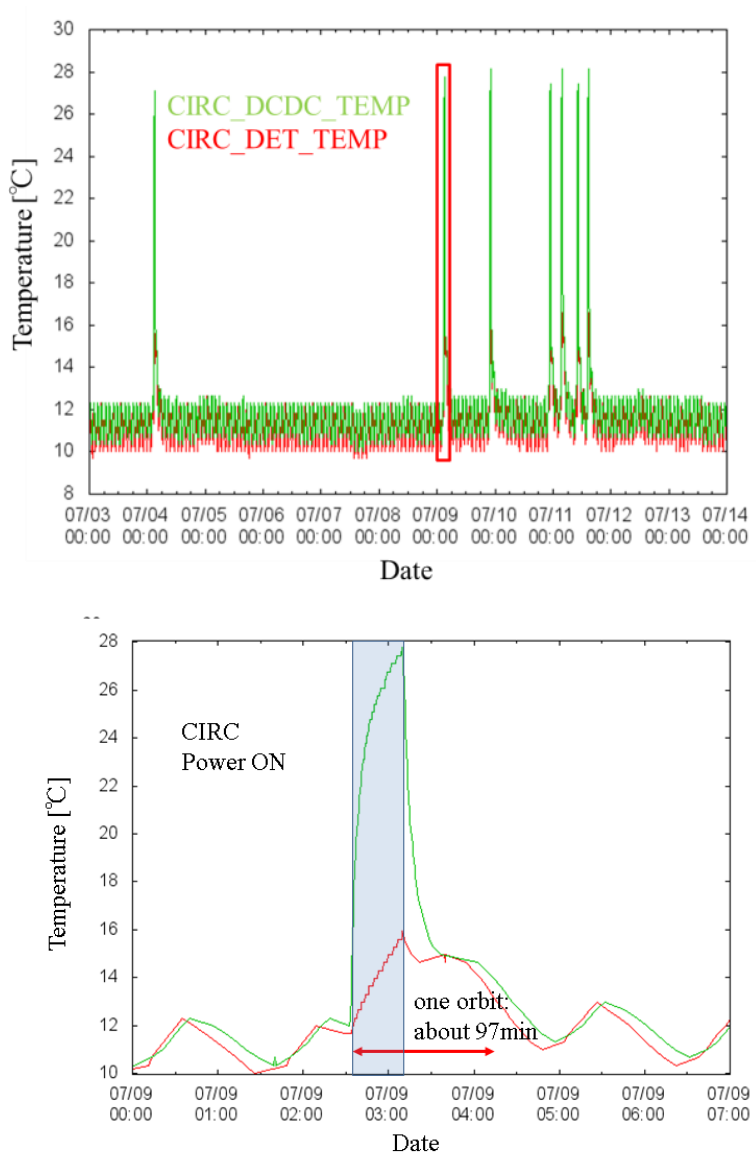

Figure 5. (Upper panel) Temperature telemetry during the initial check out. (Under panel) Extended figure of red box in the upper panel.

An image taken in the initial check-out phase is shown in Figure 6. It indicates night observation of Chugoku region and Shikoku in Japan, and represents a feature of thermal infrared image well. We also confirmed that our correction method with shutterless system was well-functioning. Figure 7 shows zoom of region A from Figure 6. As the color of an infrared image represents surface temperature, the Chugoku Mountains, such as Daisen, are shown in blue. Lake Shinji-ko and Nakaumi are also visible, and their temperatures are different from that of the Sea of Japan. Figure 8 is a zoom of region B from Figure 6. There are many islands visible in the Seto Inland Sea. The temperature distribution of the Seto Inland Sea is also apparent. Fukuyama City and Kurashiki City contain industrial areas such as steel planets and, thus, are colored yellow. Figure 9 is a zoom of region $\mathrm{C}$ from Figure 6. The Niyodo River can be seen flowing through Kochi City and mixing with the Pacific Ocean. Clouds are colored black as they are at a higher altitude than mountains.
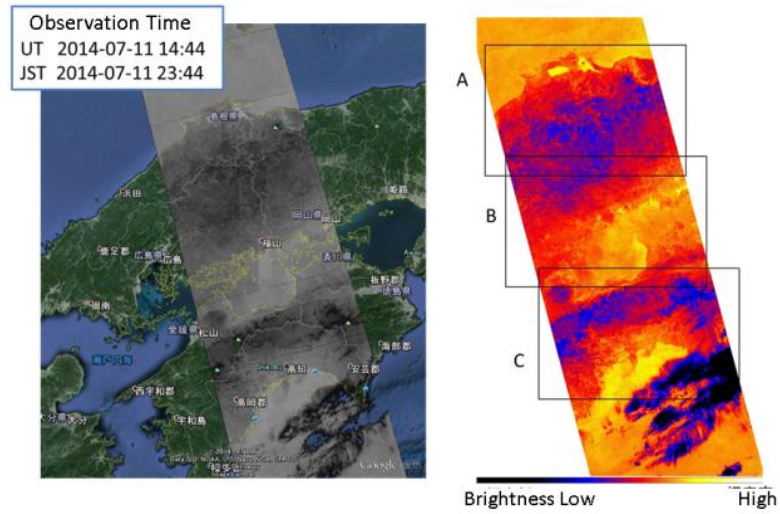

Figure 6. Night image of the Chugoku region and Shikoku in Japan (JST 23:44).

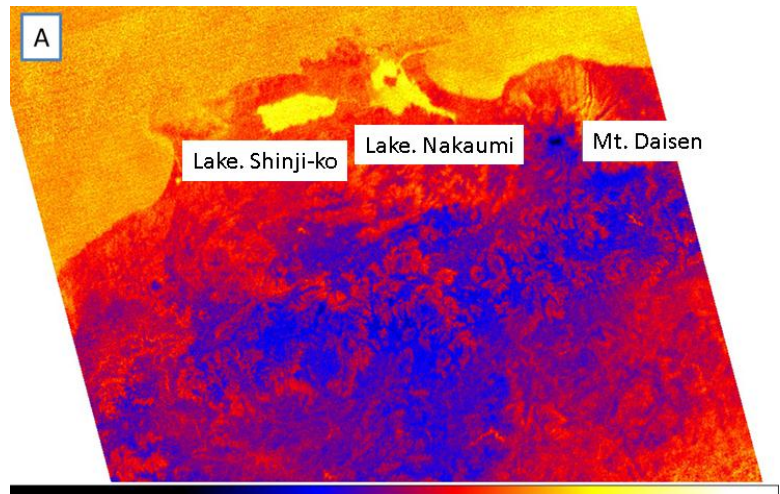

Figure 7. Zoom of the region A from Figure 6.

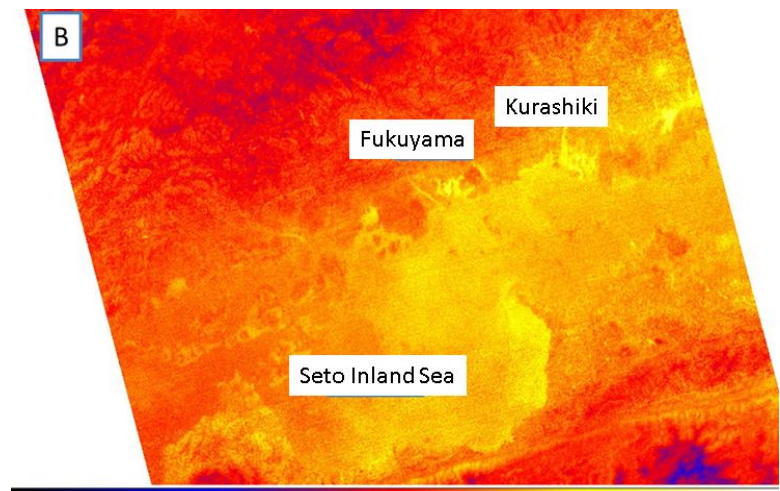

Figure 8. Zoom of the region B from Figure 6.

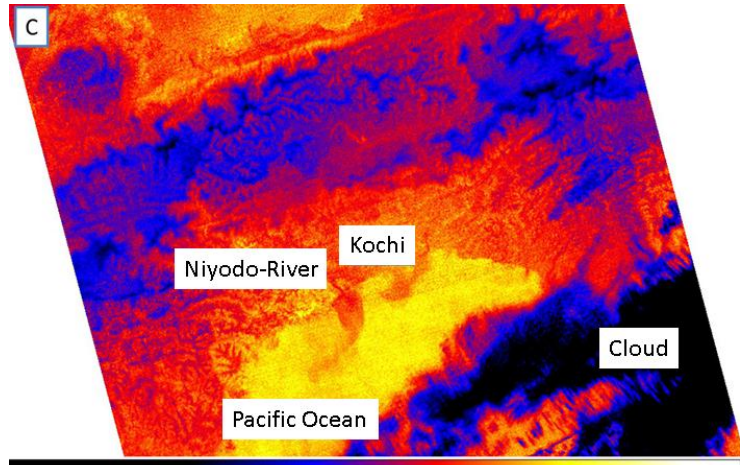

Figure 9. Zoom of the region $\mathrm{C}$ from Figure6.

Pointing determination accuracy estimated from the image shown in Figure 6 is $2.3 \mathrm{~km}$ in along track (AT) and $1.0 \mathrm{~km}$ in cross track (CT) direction. Maximum gap about $7 \mathrm{~km}$ arises 
because of its time-setting accuracy. We will reduce geometric precision error due to time lag and misalignment in the next early calibration/validation phase. In this phase, we confirmed that the ALOS-2/CIRC was properly operated as we designed.

\section{EARLY CALIBRATION/VALIDATION PHASE}

Our objectives in the early calibration validation phase are to confirm the correction method for shutterless system and absolute temperature accuracy (less than $\pm 4 \mathrm{~K} @ 300 \mathrm{~K}$ ) by vicarious calibration. Observational results are also introduced in section 5.2. We announced that ALOS-2/CIRC data is released open to the public.

\subsection{Vicarious Calibration}

Vicarious calibration is an on-orbit technique in which calibrated sensors take simultaneous measurements of a spectrally or spatially homogeneous target during periods satellite instrument overpasses. Vicarious calibration for CIRC is planned to be performed by the following three approaches.

1. Automated validation stations' data and ground experimental data

2. Airborne data with the CIRC ground test model (GTM)

3. Other satellite observation data

Vicarious calibration with the first item is conducted with a temperature-based method in which the surface temperature and spectral emissivity of a site are individually given, and used for prediction of the top of the atmosphere (TOA) radiance. In this method, atmospheric profiles (air temperature, humidity, etc.) are necessary to calculate radiative transfer. As for the second item, we carry out airborne observation using the CIRC ground test model (GTM), which is roughly similar sensor with the proto flight model (PFM) and has optical and radiometric performances equivalent to the PFM. CIRC GTM data are used for prediction of TOA radiance. In this case, atmospheric profiles are also used for radiative transfer calculation. In addition, the third item including MODIS data is carried out for cross calibration with CIRC. This paper introduces early validation results using some automated validation stations' data and ground experimental data.

In the CIRC project, a few sites such as Lake Kasumigaura, Lake Tahoe, and Lake Shinji-ko have been used for automated validation station sites. On the other hand, a ground experiment at Railroad Valley playa was conducted in the initial validation period. Figure 10 shows the relation between the original CIRC observation values calibrated with ground test results, and the predicted values in radiance and brightness temperature at TOA. The red line in each plot indicates a correction equation derived from the above relation. In the upper of Figure 11, the vicarious calibration data corrected for the CIRC brightness temperatures are shown with cross marks, indicating that the correction performs well. The lower of Figure 11 shows that the absolute temperature accuracy is expected to be within $\pm 3.3 \mathrm{~K}(3 \sigma)$. More vicarious calibration data obtained at TOA temperatures higher than 30 degree $\mathrm{C}$ are required because only one point from Railroad Valley is plotted in this temperature range at present. In the next phase, we aim at temperature accuracy less than $\pm 2 \mathrm{~K} @ 300 \mathrm{~K}$ by ground-based measurement in Lake Kasumigaura and airborne observation with CIRC GTM.
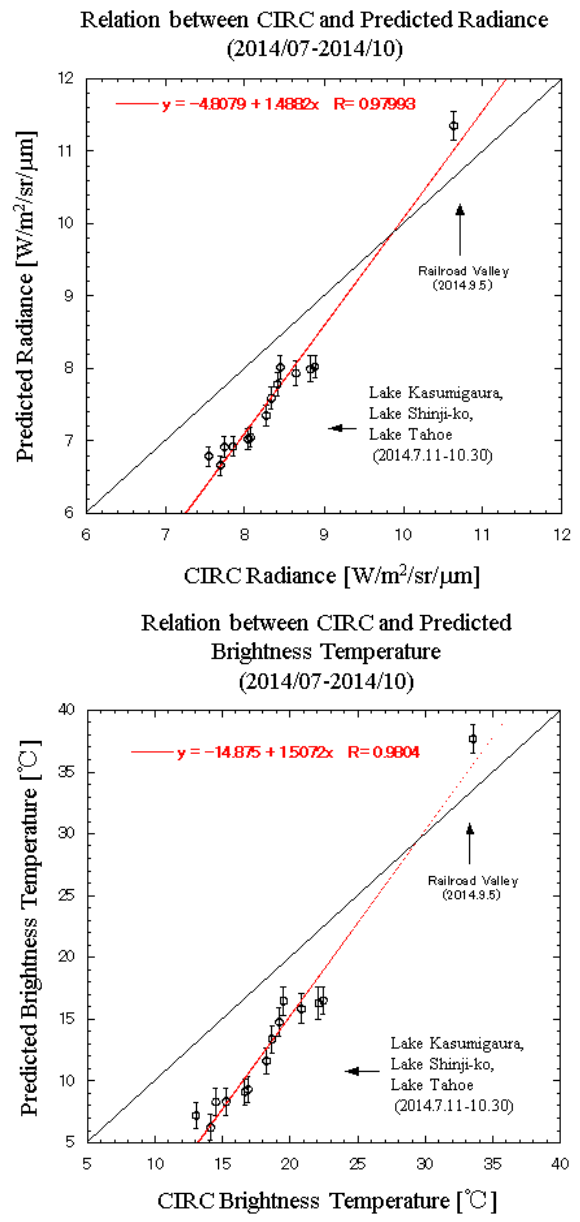

Figure 10. Relation between observed CIRC data and predicted values about Radiance and Brightness Temperature.
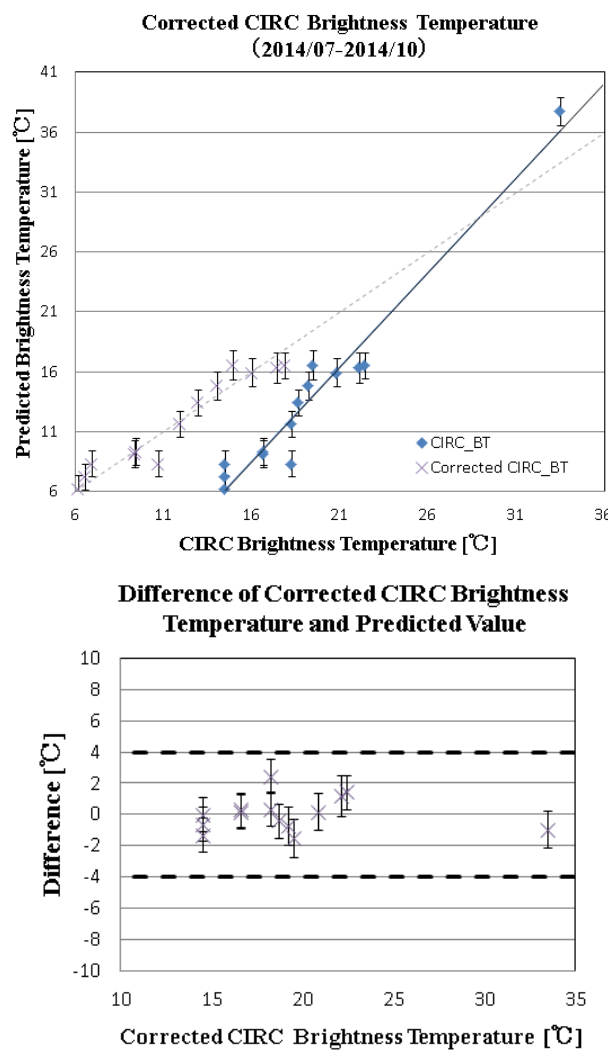

Figure 11. Corrected CIRC Brightness Temperature 


\subsection{Observational Results about Three Missions}

This section shows observational results about three missions observed based on CIRC basic observation plan.

5.2.1 Wildfire: Wildfires are detected more than 2800 scenes including thermal anomaly of volcanoes from the initial check-out phase to January 2015 (see Figure 12). Figure 13 shows detected wildfire in Angola. Yellow regions of the CIRC image, which indicate high temperature, are consistent with results of wildfire detection by MODIS

\section{Fire Detected}

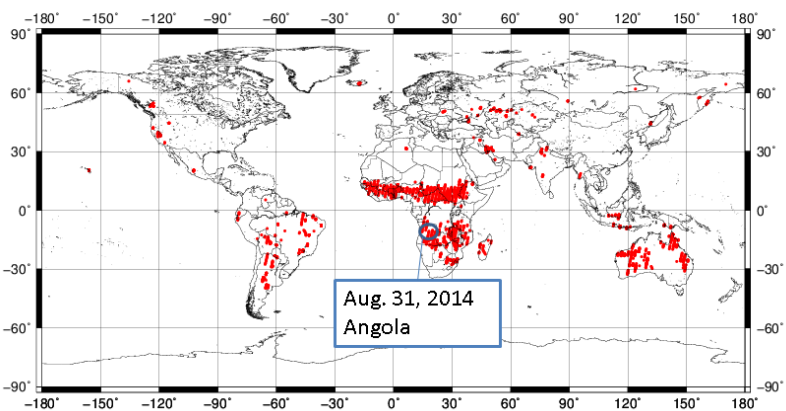

Figure 12. Fire detected (during July 2014 to January 2015).
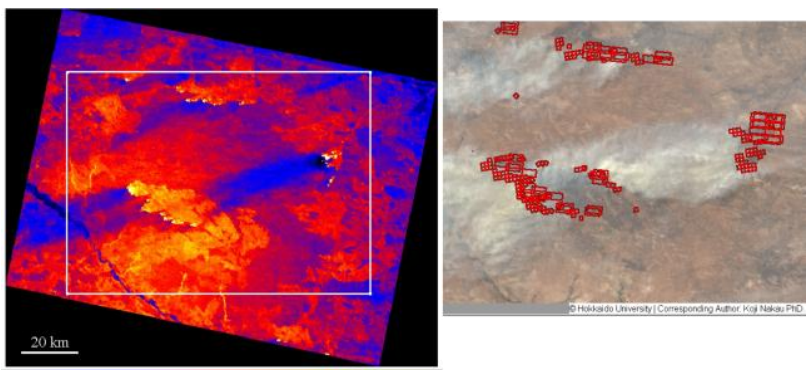

Figure 13. Detected wildfire in Angola (left panel) on August 31 at UTC 10:29. Right panel shows MODIS visible image. Red areas are wildfire detected (MOD14) provided by Nakau.

5.2.2 Volcanoes: Image of Bardarbunga volcano in Iceland is shown in Figure 14. Temperature is high in an order of white, yellow, red, blue, and black. Region in an elongated hightemperature shape is seen. As shown in this figure, lava flowed from the volcano toward northeast, and attained the length of 20 $\mathrm{km}$. Even high-temperature region can be clearly seen because dynamic range of the CIRC is broad.

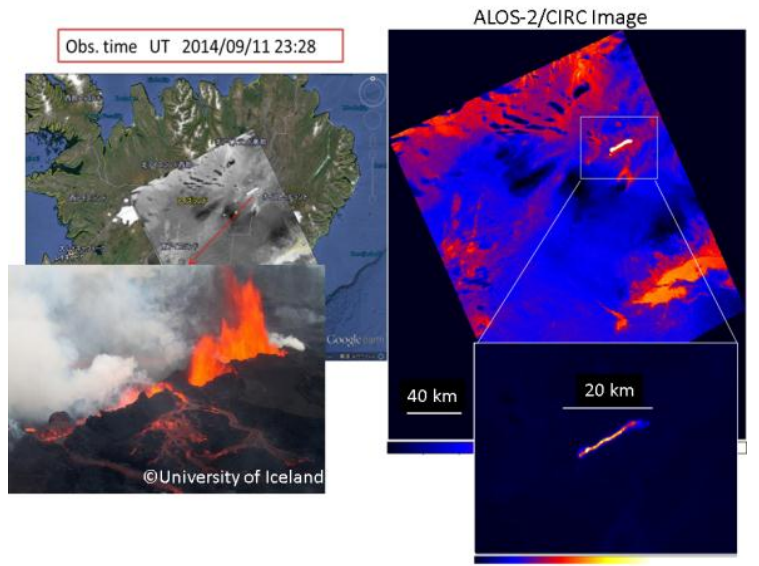

Figure 14. Bardarbunga volcano in Iceland (UTC 23:28, September 11, 2014).
Figure 15 shows a result of Nishi-no-shima, which is a volcanic island in Japan. An eruption created a new island southeast of the volcano in November 2013. The active eruption continued into 2015. As shown in Figure 15, high-temperature region is thought to be extended lava flow.

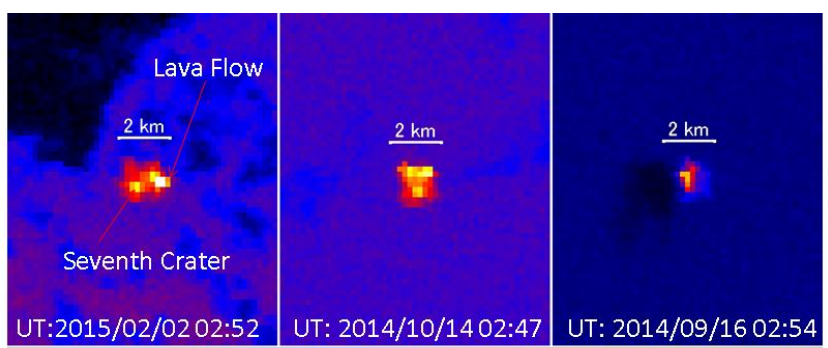

Figure 15. Nishi-no-shima in Japan.

5.2.3 Urban heat Island: Figure 16 shows Chicago observed on July 21 at CST 13:40. As the land-water temperature difference is larger during daytime than during nighttime observation, a coast line is clearly visible. Figure 17 shows a zoom of white box from Figure 16. A grid of streets and avenues is visible around the city. Despite the relatively large spatial resolution of CIRC (200 m) compared to the width of roads $(<60 \mathrm{~m})$, the road pattern is still apparent due to the stark temperature difference between the roads and other surroundings areas.

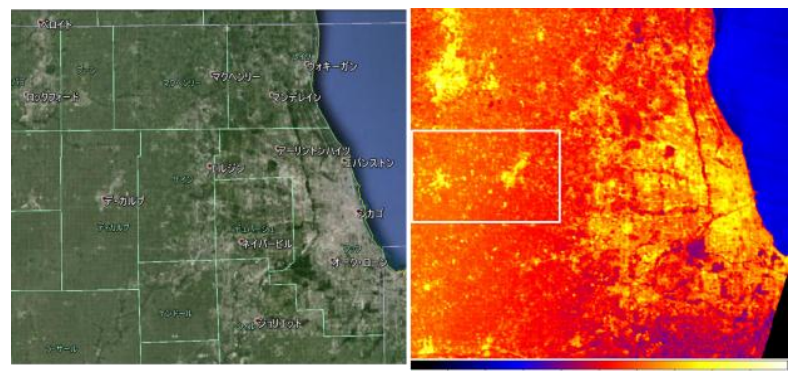

Figure 16. Daytime image of Chicago in the stats of Illinois (CST 13:40, UTC 18:40, July 21, 2014).

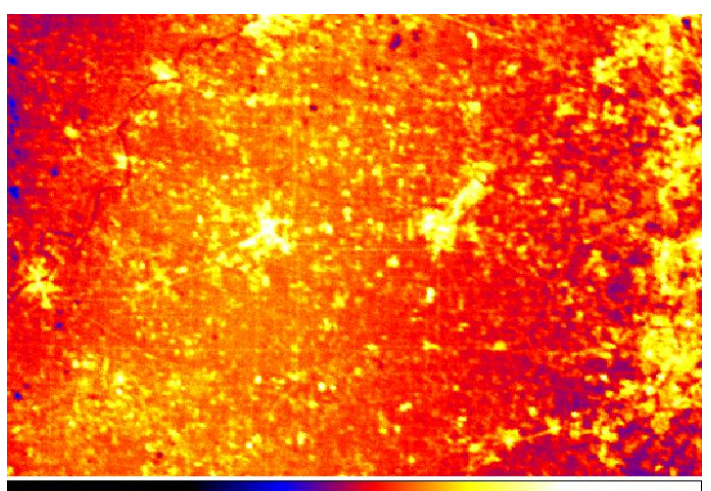

Figure 17. Zoom of white box from Figure 16.

5.3 Data release to the Public about ALOS-2/CIRC observation data

After the calibration and validation of ALOS-2/CIRC and data quality has been confirmed to be adequate, ALOS-2/CIRC data is now open to public. All ALOS-2/CIRC data is available from CIRC observation data search (see Figure 18), if user follows the CIRC data policy. All user should show (C) JAXA in the image of CIRC. 


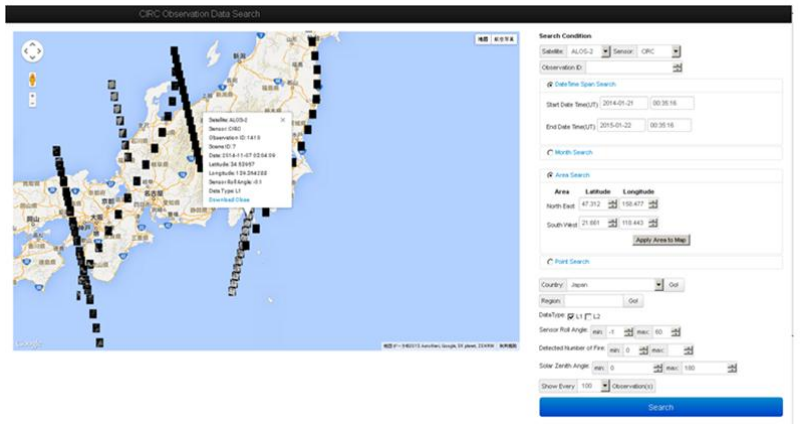

Figure 18. Example of CIRC observation data search

\section{SUMMARY}

CIRC is the first microbolometer sensor without a calibration function for space application, and achieves a small size, light mass, and low electrical power consumption by employing athermal optics and a shutterless system. We confirmed CIRC functional and performance verification onboard ALOS-2 in the initial check-out phase. We have also confirmed the correction method with shutterless system, and verified absolute temperature accuracy of less than $\pm 4 \mathrm{~K} @ 300 \mathrm{~K}$ by vicarious calibration in the early calibration validation phase. The CIRC has acquired the images of Earth actually about wildfires, volcanoes, and urban heat island since the initial check-out phase. ALOS-2/CIRC data has been available to the public.

\section{REFERENCES}

CIRC team, "CIRC Observation Data Search", http://circgs.tksc.jaxa.jp/data/index.html

Katayama, H., Okamura, Y., Tange, Y., and Nakau, K., 2008. Proc. ISTS, n-30p.

Katayama, H., Naitoh, M., Suganuma, M., Harada, M., Okamura, Y., Tange, Y., and Nakau, K., 2009. Development of the Compact Infrared Camera (CIRC) for Wildfire Detection. Proc. SPIE, vol 7458, pp. 745806-1-745806-8.

Kato, E., Katayama, H., Naitoh, M., Harada, M., Nakamura, R., and R. Sato, R., 2012. Development of the Compact Infrared Camera (CIRC) for earth observation. Proc. SPIE, 8353, 8353J1.

Kato, E., Katayama, H., Naitoh, M., Harada, M., Nakamura, R., Nakau, K., Tange, Y., and Sato, R., 2014. Radiometric Calibration of Compact Infrared Camera (CIRC) for Earth Observation. Sensors and Materials, 26, No.4, pp. 199-214.

Nakamura, R., Katayama, H., Naitoh, M., Harada, M., Kato, E., Nakau, K., and Sato, R., 2013. Ground Calibration of Compact Infrared Camera (CIRC) for Earth Observation. Proceedings of 2013 IEEE International Geoscience and Remote Sensing Symposium, pp. 4435-4438.

Nakau, K., 2014. "Wildfire Database", http://fire.cris.hokudai.ac.jp.

Suzuki, S., Osawa, Y., Hatooka, Y., Kankaku, Y., and Watanabe, T., 2009. Overview of Japan's Advanced Land Observing Satellite-2 Mission. Proc. SPIE, vol 7474, pp. 7474Q-1-10.
Torii, S., et al, 2008. CALET mission on ISS. Proc. SPIE, vol 7021, pp. 702114-11. 